\title{
Perioperative Takotsubo Cardiomyopathy (Broken Heart Syndrome)-A Diagnostic Dilemma
}

\author{
S. Kiran ${ }^{1}$ Shalendra Singh ${ }^{1} \quad$ Nipun Gupta ${ }^{1}$ Deepak Dwivedi ${ }^{1} \quad$ Kaminder Bir Kaur $^{1}$
}

\author{
${ }^{1}$ Department of Anaesthesia and Critical Care, Armed Forces \\ Medical College, Pune, India
}

J Neurosci Rural Pract 2020;11:355-356

Takotsubo cardiomyopathy (TCM) is a stress-induced cardiomyopathy (apical ballooning syndrome/broken heart syndrome) triggered by an acute medical illness or intense physical or emotional stress. ${ }^{1}$ It is characterized by acute-onset symptoms associated with electrocardiographic (ECG) abnormalities suggesting an acute coronary syndrome in the setting of absence of obstructive coronary artery disease. ${ }^{1}$ TCM has been classified based on the etiology into primary and secondary subtypes, with structural brain damage and anesthetic stress being common triggering factors for secondary TCM. ${ }^{2}$ We describe a patient who developed secondary TCM postoperatively after evacuation of chronic subdural hematoma under general anesthesia (GA).

A 77 -year-old man $(160 \mathrm{~cm}, 70 \mathrm{~kg})$, a known case of hypertension on regular medications, presented with a history of fall at his residence 2 months back followed by gradually progressive continuous headache and imbalance while walking. He also experienced memory disturbances after 1 month of the fall. Patient gave a history of aggravation of all his symptoms for the past 5 days with no associated history of vomiting, seizure, weakness of limbs, or sphincter disturbances. Examination revealed a Mini-Mental State Examination score of 28/30 and ataxic gait. On investigation, hemogram and biochemistry were within normal limits and noncontrast computed tomography of head revealed bilateral frontotemporal-parietal subdural hematoma. Patient was planned for bilateral frontal and parietal burr holes with evacuation of chronic subdural hematoma. Surgery was conducted under GA with invasive blood pressure monitoring and central venous access via right internal jugular vein. Surgery was uneventful with evacuation of 60 to $75 \mathrm{~mL}$ dark colored hematoma bilaterally. However, patient developed hypotension at the end of the surgery and was supported with infusion noradrenaline to maintain a mean arterial pressure of $65 \mathrm{~mm} \mathrm{Hg}$. In view of poor respiratory effort and ongoing hypotension, patient was not extubated and shifted to intensive care unit for elective mechanical ventilation and further management. Patient was sedated and intensive care support continued as per the institution protocol. Six hours
Address for correspondence Shalendra Singh, DM, Department of Anaesthesiology and Critical Care, Armed Forces Medical College, Pune 411040, India (e-mail:drsinghafmc@gmail.com).

in the postoperative period, patient had persistent hypotension and developed tachycardia in spite of infusion noradrenaline at $0.6 \mu \mathrm{g} / \mathrm{kg} / \mathrm{min}$. In view of the above, vasopressin was added at $0.04 \mathrm{U} / \mathrm{min}$. On first postoperative day, patient remained tachycardic and hypotensive with continued vasopressor support, in spite of adequate fluid resuscitation. Arterial blood gas picture revealed severe metabolic acidosis with lactates of $4.1 \mathrm{mmol} / \mathrm{L}$. Simultaneously, patient developed increased ventilatory requirement with a positive end expiratory pressure of $14 \mathrm{~cm} \mathrm{H}_{2} \mathrm{O}$ and $\mathrm{FiO}_{2}$ of 0.8 . ECG done on first postoperative day showed sinus tachycardia with no ST-T changes and chest roentgenogram showed increased bronchovascular markings. In view of worsening clinical situation and to look for any cardiac cause, a bed side two-dimensional transthoracic echocardiography (ECHO) was done which showed apical ballooning with hypercontractile basal septum with a left ventricular ejection fraction of 35\% (-Fig. 1). In view of recent intracranial surgery, high dose

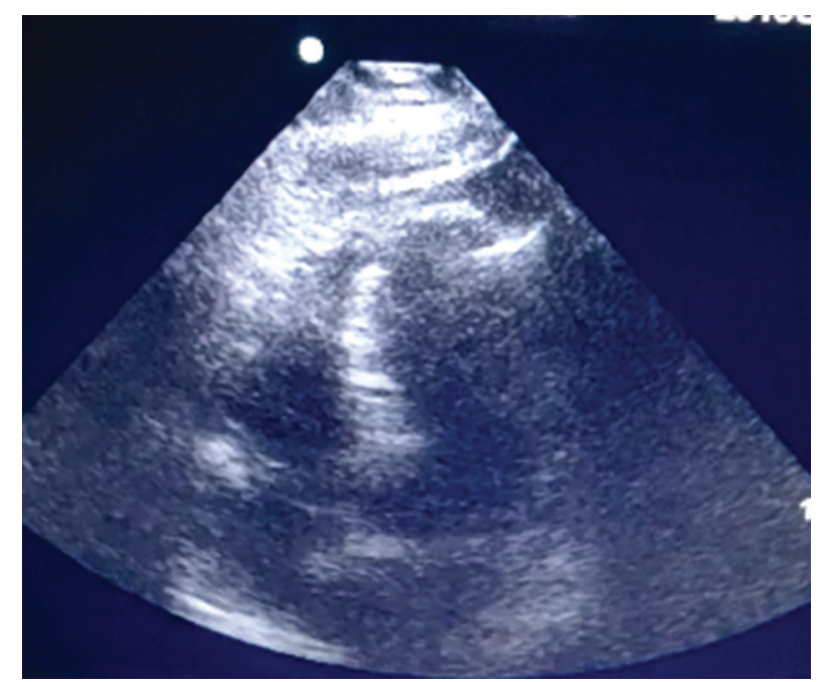

Fig. 1 Bed side two-dimensional transthoracic echocardiography showing cardiac apical ballooning with hypercontractile basal septum. 
of vasopressors and corroborative ECHO findings, TCM was confirmed and patient was planned for conservative management with a plan to decrease vasopressors and increasing intravenous fluids. On third postoperative day, patient continued to deteriorate with requirement of high vasopressor and ventilatory support associated with progressive fall in urine output. Patient further deteriorated and had a hypotensive cardiac arrest from which he could not be revived.

It is emphasized that TCM is not an uncommon clinical entity and can be a life-threatening event, hence, should be considered as a differential diagnosis in any patient who decompensates in the perioperative period. Being a reversible condition, early diagnosis and appropriate management with vasopressors and fluids are the key to successful outcome.

\section{Funding}

None.

\section{Conflict of Interest}

None declared.

\section{References}

1 Agarwal S, Bean MG, Hata JS, Castresana MR. Perioperative takotsubo cardiomyopathy: a systematic review of published cases. Semin Cardiothorac Vasc Anesth 2017;21(4):277-290

2 Komamura K, Fukui M, Iwasaku T, Hirotani S, Masuyama T. Takotsubo cardiomyopathy: pathophysiology, diagnosis and treatment. World J Cardiol 2014;6(7):602-609 